\title{
Valvular Regurgitation by Color Doppler Echocardiography
}

\author{
A ndré Schmidt, O swaldo C. Almeida Fon, A ntonio Pazin Fo, José A. Marin-N eto, Benedito C. Maciel
}

Ribeirão Preto, SP - Brazil

\section{Overview}

Precise characterization of the magnitude of valvular regurgitations and of the functional repercussions associated with them still remains one of the major challenges in modern clinical cardiology. Determination of the degree of hemodynamic overload imposed on the heart resulting from valvular regurgitations is fundamental to defining the best moment for indicating surgical interventions in such clinical conditions. Identification of the best moment for surgery in this type of valvular disease depends on the characterization of 2 opposite prognostic factors: on the one hand, evaluation of immediate and late risks of the different types of procedures available; on the other hand, the potential implications for left ventricular function caused by an occasional delay in this therapeutical indication.

The ideal method for precise quantification of valvular regurgitations should comprise the following: 1) the ability to measure the regurgitant flow or fraction or the dimensions of the regurgitant orifice, or both; 2) the possibility of quantitative assessment of hemodynamic repercussions of valvular regurgitation on left ventricular function and pulmonary circulation; 3 ) to allow comparisons over time; 4) to be as least invasive as possible; 5) to have a low operational cost.

In the clinical context, significant limitations in the different methods of quantifying valvular regurgitations have been reported ${ }^{1,2}$. Angiocardiography with iodine contrast media ${ }^{3-5}$ was the first quantification method that ended up being consolidated over the years as a reference pattern. This technique, however, in addition to its invasive character, which is a limitation to its serial use, has additional significant problems. While its use in the semiquantitative way has a significant subjective component, which makes it extremely dependent on the observer's experience, the described quantitative ways of using this method for the calculation of the regurgitant fraction have variations ranging from

Faculdade de Medicina de Ribeirão Preto - USP

Mailing address: Benedito Carlos Maciel - Divisão de Cardiologia - Departamento de Clínica Médica - Faculdade de Medicina de Ribeirão Preto-USP -

14049-900 - Ribeirão Preto, SP, Brazil
$5 \%$ to $15 \%{ }^{6}$. In addition, technical factors, such as the intensity of fluoroscopy, the use of one or two planes for obtaining the images, the volume of the contrast medium used and the moment of its injection, the position of the catheter tip and its type, previous injections of contrast medium, heart rate in the different phases of the exam, dimensions of the receiving chamber, the presence of associated valvular lesions, and the occurrence of ectopic beats, impose a considerable limitation on this methodology ${ }^{7}$.

Use of the Doppler technique with two-dimensional echocardiography inspired new perspectives for new metho$\mathrm{ds}$ for quantifying valvular regurgitation. Among the undeniable advantages of this methodology, we can cite its noninvasive characteristic, which allows serial and comparative assessments of the same patient, and its wide availability and relatively low cost. At the same time, in addition to allowing grading of the magnitude of the valvular regurgitation, it also allows the satisfactory quantification of functional repercussions of valvular lesion upon left ventricular function and pulmonary circulation. Some significant limitations should be mentioned: 1 ) the method depends on the existence of an ultrasound window adequate for obtaining high-quality two-dimensional images and Doppler signs with a low level of interference; 2) the operator's experience in the obtainment and interpretation of the images has a significant influence on the quantification provided by the method.

Thanks to the already mentioned qualities and the enormous technological development, which in addition to providing obtainment of two-dimensional images with high-quality spatial and temporal resolution also has allowed the progressive incorporation of new forms of representation of the Doppler sign - evolving from the spectral Doppler (pulsed and continuous) to the Doppler color flow mapping - Doppler echocardiography has acquired an important role in the clinical assessment of patients with valvular regurgitation. At the same time, it has become a resource of quantitative investigation of cardiovascular function and mechanisms determining valvular dysfunction associated with various clinical conditions. This methodology has shown in comparative studies of spectral Doppler alone with contrast angiography mean levels of sensitivity of $90 \%$ for mitral regurgitation and of $95 \%$ for aortic regurgitation, as well as specificity of $93 \%$ and $96 \%$, respectively ${ }^{8}$. Addition of color flow mapping increased sensitivity and speci- 
ficity, with specificity reaching levels of up to $100 \%$ for mitral and aortic regurgitation. With color flow mapping, this technique may identify minimum degrees of regurgitation in a significant proportion of healthy individuals ${ }^{9,10}$. Especially in this group of individuals, color flow mapping managed to show that the pulsed Doppler signs verified close to normal valves and initially interpreted as resulting from a volume of valvular closure ${ }^{11,12}$, effectively represented a true regurgitation ${ }^{13}$. In the healthy population, tricuspid and pulmonary regurgitations have been reported in up to $83 \%$ and $88 \%$, respectively, but the occurrence of mitral and aortic regurgitations are less frequent (up to $48 \%$ and $33 \%$, respectively) ${ }^{14}$. Particularly in older age groups, the prevalence of mitral and aortic regurgitations may reach $71 \%$ and $89 \%$, respectively ${ }^{15-17}$.

\section{Doppler Spectral Analysis}

Qualitative and semiquantitative methods - The first attempts to use the Doppler technique for quantifying mitral valve regurgitation were conducted with the pulsed method ${ }^{18}$, mapping with the Doppler sample from the valvar plane the entire extension of the left atrial cavity and defining the severity of the regurgitation in 3 levels, according to the extension of the signal of the regurgitant flow in that chamber. This approach is based on the conception that the extension of the flow disturbance documented through Doppler signals in the atrial cavity reflects the regurgitant volume. The correlation observed with angiography with iodine contrast in the first studies was fairly satisfactory $(\mathrm{r}=0.88)$. However, more modest values of correlation were documented in further investigations ${ }^{19,20}$. This same technique when applied to patients with aortic regurgitation ${ }^{21,22}$ showed similar results. This method, however, is difficult to perform because it takes an excessive amount of time, which, from the beginning, has imposed limitations on its clinical application. Especially in aortic regurgitation, it is practically impossible to adequately characterize the dispersion of the regurgitant flow because of the mitral diastolic flow that enters the left ventricle in the same direction as the aortic regurgitant flow. In addition, flows that adhere to the walls of the receiving chamber impose another important limitation, making detection and follow-up of the intrusion extremely difficult ${ }^{23}$. These limitations and the further appearance of Doppler color echocardiography have substantially reduced the importance of this quantifying method.

A qualitative approach of the severity of mitral regurgitation was described based on the analysis of the intensity of the continuous-wave Doppler signals ${ }^{24}$. This approach was based on the direct plausible relation between the intensity of the Doppler signal and the regurgitant volume. Therefore, considering the visual comparison between the intensity of the anterograde and regurgitant flow signals through the mitral valve, it is possible to characterize regurgitation as of a mild degree if the signal is less intense when compared with that of the anterograde flow. On the other hand, valvular regurgitations involving large regurgitant volumes tend to show intensity similar to that of the Doppler signal corresponding to the retrograde and anterograde flows, independently of adjustments of gain in the ultrasound system, because both signals are represented in the same image. The profile of the range of velocity (envelope) of the regurgitant flow on continuous-wave Doppler is another qualitative method that has been described ${ }^{25}$ for assessing the severity of mitral valve regurgitation. In more severe valvular regurgitations, a delay in the reduction in the velocity of the end-systolic phase has been reported. These techniques have a limited clinical applicability because of their qualitative character and small capacity for differentiating the severity of regurgitation. A recent study ${ }^{26}$ using computed analysis of the intensity of the Doppler signal for quantifying valvular regurgitation identified several factors, in addition to the regurgitant volume, that could interfere with the intensity of signal. These factors include imperfect alignment between flow and sample directions, such as occurs in eccentric jets, instrumental adjustments, and hemodynamic factors. Furthermore, based on the comparison between the visual and the computed analysis, the need for altering at least $20 \%$ of the intensity of the signal was observed so that the signal could be detected in the visual analysis. Yet in regard to mitral regurgitation, the pulsed Doppler technique coupled with identification of the pulmonary vein flow through color flow mapping made possible the documentation of the pulmonary vein flow pattern using transesophageal or even transthoracic transducers. This pulmonary vein flow pattern clearly adjusts when a significant increase in the left atrial pressure occurs. Therefore, in severe mitral valve regurgitations, a reversion of the systolic flow in the pulmonary veins occurs, showing a high specificity for that diagnosis varying from $80 \%$ to $100 \%{ }^{27-29}$.

In aortic regurgitation, during systole, the flow between the aorta and the left ventricle depends directly on the dimension of the regurgitant orifice. In the same way, the velocity with which the pressure gradient between these two chambers decreases during diastole also varies directly with the magnitude of the valvular reflux. Therefore, analysis of the inclination of the diastolic velocity of the aortic flow reflects the velocity of reduction in the aorta/left ventricle gradient and, consequently, the dimensions of the regurgitant orifice. Mild valvular regurgitations show a slow decline in the diastolic velocity, usually lower than $1.5 \mathrm{~m} / \mathrm{s}$, but severe regurgitations show a rapid decline, with values higher than $3 \mathrm{~m} / \mathrm{s}^{30}$. Even though reasonable levels of correlation have been observed between these 2 indices with the invasive measure of the regurgitant fraction ${ }^{31}$, a significant overlap occurred between the diverse groups of patients classified based on angiographic grading.

Flow in the initial portion of the descending aorta is mainly systolic, even though a discrete retrograde flow may be identified at the beginning of diastole. In patients with aortic regurgitation, this retrograde component of the diastolic flow becomes more intense. Several attempts to calculate the regurgitant fraction were performed based on the quantitative relation between these systolic and diastolic components of 
the aortic flow velocity. Difficulties related to the existence of a small retrograde flow in healthy individuals and the variability of the aortic dimensions during the cardiac cycle limited the quantifying capacity of this method and, therefore, its clinical use. A simpler variant of this technique identifies, with the pulsed Doppler, the retrograde flow in the abdominal aorta, which does not happen in the healthy individual. Therefore, presence of retrograde flow during the entire diastole allows the identification of patients with severe aortic regurgitation, but in patients with mild to moderate lesions, the reverse flow is only documented in the initial phase of diastole. This technique, even though simple and only semiquantitative shows a good correlation with the semiquantitative angiographic grading ${ }^{32}$.

\section{Quantification of the volume and the regurgitant fraction}

Direct quantification of the flow and of the regurgitant fraction may be performed with the pulsed Doppler technique. Based on the principle that the flow in a circulatory segment may be calculated as the product between the integral of the velocity documented by Doppler and the sectional area of this segment, the transvalvular anterograde flows may be calculated. In patients with mitral regurgitation, the regurgitant flow may be calculated as the difference between the mitral diastolic flow, which is equivalent to the systemic anterograde flow plus the regurgitant flow and the systolic flow measured through the aortic valve. In patients with aortic regurgitation, the regurgitant flow may be quantified by the difference between the aortic transvalvular systolic flow, which represents the sum of the systemic and regurgitant flows, and the systemic flow measured through the mitral or pulmonary valves. The regurgitant fraction may be obtained by dividing the regurgitant flow by the systemic anterograde flow. Even though this technique may be considered well validated based on the consistency of published clinical and experimental results ${ }^{33,34}$, its clinical application is extremely limited. This is mainly due to the fact that this technique requires an excessively long time for data collection. In addition, some limitations of this methodology may be stressed as follows: 1) the multiple steps required to calculate the different flows and area measurements, which involve dimensions to the power of 2 , increase the probability of errors; 2) the variables used are not simultaneously measured; 3) avoidance of underestimation of transvalvular flow velocities depends on a high quality Doppler signal; and finally, 4) the impossibility of using this technique in the presence of other associated valvular regurgitations.

\section{Color flow mapping}

The technological development allowing the appearance of Doppler color flow mapping opened new possibilities for a more precise quantification of the magnitude of the regurgitant flow. Since the beginning, the high sensitivity demonstrated by this technique for identifying the presence of minimum intracardiac flow disturbances was evident. In the context of valvular regurgitations, it was possible to document in a very simple way and in real time, with acceptable levels of temporal resolution, the extension of this disorder in the receiving chamber without the tedious and long-lasting processing required by the pulsed spectral method. At the same time, it was possible to document the spatial distribution of the flow close to the regurgitant orifice.

\section{Dimensions of the regurgitant jets}

The initially proposed techniques for estimating the severity of valvular regurgitations involving color Doppler echocardiography were based on the assumption that the spatial distribution of the jet velocities would reflect the regurgitant volume. The initially published studies ${ }^{35-39}$ related to quantification of mitral valve regurgitation, even though showing satisfactory levels of correlation (varying from 0.76 to 0.87 ) between the dimensions of the regurgitant jet and the semiquantitative angiographic assessment, showed a significant dispersion. On the other hand, the correlation between the measurements of the maximum jet area and the regurgitant volume or fraction was very limited ( $r=0.55$ and $r=0.62$, respectively). In the clinical context, it was observed that jets with maximum areas above $8 \mathrm{~cm}^{2}$ showed sensitivity and specificity of $82 \%$ and $94 \%$, respectively, for identifying severe mitral valve regurgitations. On the other hand, maximum jet areas lower than $4 \mathrm{~cm}^{2}$ predicted mild valvular regurgitation with sensitivity of $85 \%$ and specificity of $74 \%$, when compared with angiography. Between these 2 limits were mainly the patients with moderate regurgitation on angiography, even though a significant superposition with other degrees of reflux was reported.

Aiming to overcome these limitations, to quantify mitral regurgitation Helmcke et al ${ }^{38}$ proposed the analysis of jet dimensions using more than one plane for acquiring images. They also proposed the grading of valvular reflux according to the relation between the areas of the regurgitant jet and the left atrium. With this approach, these investigators reported an improvement in the indices of correlation with contrast angiography and smaller data dispersion. They obtained a better distinction between patients, which were angiographically classified into 3 levels of severity, and reported concordance between the 2 methods in $94 \%$ of the cases. Therefore, regurgitant jet areas smaller than $20 \%$ of the atrial area were classified as mild degree, showing a positive predictive value, sensitivity, and specificity of $100 \%, 94 \%$, and $100 \%$, respectively. Regurgitant jet areas between $20 \%$ and $40 \%$ of the left atrial area were consistent with moderate mitral regurgitation, with diagnostic power of $85 \%, 94 \%$, and $95 \%$, respectively, for positive predictive value, sensitivity, and specificity. Finally, maximum regurgitant jet areas higher than $40 \%$ of the left atrial area were classified as severe regurgitation showing a positive predictive value, sensitivity, and specificity of $93 \%$, $93 \%$ and $96 \%$, respectively. In spite of these data, controversy ${ }^{39,40}$ in regard to effectiveness of this relation with the 
atrial area exists, because proportionality between the size of the jet and the receiving chamber does not have a physiological base. In addition, even though the correlation improves with normalization for the atrial area, this variation is not statistically significant.

Another study ${ }^{41}$ confirmed the improvement in the correlation between jet dimension and angiographic quantification after normalization for the left atrial area in mitral regurgitation and for the dimension of the left ventricular outflow tract in patients with aortic regurgitation. However, the rheumatic cause reduces the capacity of differentiating between the diverse degrees of regurgitation defined by angiographic classification and, despite the elevated levels of correlation between the 2 methods, a significant superposition was identified, especially between the mild and moderate degrees of regurgitation.

Perry et al ${ }^{42}$ studied patients with aortic regurgitation and proposed a relation between the cross area of the jet and the area of the left ventricular outflow tract as a variable representing the severity of valvular reflux. Therefore, they obtained better levels of correlation with the angiographic grading and substantially reduced the superposition between the different degrees of valvular regurgitation. A relation between the jet area and the outflow tract area in the minor axis higher than $60 \%$ indicated severe aortic regurgitations, while values below $25 \%$ indicated mild regurgitations, when compared with angiography with iodine contrast. This same study analyzed the value of the aortic regurgitant jet diameter close to the aortic valve in the parasternal longitudinal view as an index for quantifying reflux severity. In the same way, these authors reported an improvement in the correlation with the angiographic grading $(r=0.78$ to 0.91$)$ when they began to correct that variable dividing it by the dimension of the outflow tract at the same place. Therefore, in patients with severe aortic regurgitations on angiography this relation was greater than $65 \%$, but in those patients with mild regurgitation the values were lower than $46 \%$.

All together, these clinical data show that the analysis of the spatial distribution of regurgitant flow velocities in the receiving chamber provides, at most, a semiquantitative assessment of the magnitude of valvular regurgitations. At the same time, that analysis does not allow an absolute distinction between the groups classified on angiography with iodine contrasts, which is a method that also has significant intrinsic limitations.

A more precise understanding of the multiplicity of the factors involved in determining the dimension of regurgitant jets, as well as of the complex relations existing between these factors, has been simultaneously established based on different animal and in vitro experimental studies, where the conditions of investigation were standardized in order to isolate the effect of these multiple determinants. It has also become evident that flow disturbance in the receiving chamber, which determines variations in blood velocity that constitute the base for their detection on Doppler technique, did not depend only on the blood volume crossing the regurgitant orifice. Flow disturbance also depends on blood present in the receiving chamber, which mixes with the regurgitant flow during its intrusion and then becomes part of the jet on color flow mapping. This phenomenon is called jet entrainment ${ }^{40}$. At the same time, several instrumental, physical, and hemodynamic variables influencing the magnitude of regurgitant jets, such as documented on color Doppler echocardiography, have been identified.

The specific characteristics of signal processing in each ultrasound system are among the instrumental factors, including particularities of the color algorithms, which are not standardized in the equipment industry. Consequently, assessment of the regurgitant jet magnitude involving one patient studied with different pieces of equipment may result in significant discrepancies ${ }^{43,44}$. In addition, the color gain, the frequency of pulse repetition, and the frequency of the transducer used ${ }^{45-47}$ also influence jet dimensions (fig. 1). A direct relation between regurgitant jet dimensions and color gain in the ultrasound system may occur. On the other hand, an inverse relation between jet size and the frequency of pulse repetition or the frequency of the transducer used occurs. Therefore, the clinical implications of these observations are obvious: for adequate comparison between studies performed under diverse conditions, standardization of the equipment and constancy of the parameters of imaging acquisition are essential.

In addition to the regurgitant flow, a set of interrelated physical and hemodynamic variables influences spatial dispersion of the regurgitant jets, as documented by the color Doppler technique, and includes the following: dimension of the regurgitant orifice, the pressure gradient through this orifice, and the pressure in the receiving chamber ${ }^{48,49}$. The pressure gradient through the orifice seems to be the most important factor for determining jet dimensions ${ }^{48}$, and it has a direct relation with the jet area. Progressively higher regurgitant flows are required to maintain the same degree of spatial jet distribution as dimensions of the regurgitant orifice increase. For a given orifice, a direct relation between regurgitant flow and jet area may be documented. On the other hand, if the regurgitant flow is kept constant, an increase in orifice dimensions is followed by a reduction in jet dimensions.

Maciel et $\mathrm{al}^{40}$ studied in an in vitro experimental model the effects of pressure and volume of the receiving chamber on the dimensions of the regurgitant jets and documented that, if the regurgitant volume and the receiving chamber volume were kept constant, the jet area diminished with the increase in pressure in that cavity. Therefore the pressure/ volume relation inside the receiving chamber seems to be an important and independent factor in determining the spatial distribution of these jets. These experimental data allow understanding the clinical observations by Harlamert et al ${ }^{50}$ who have reported patients with comparable degrees of mitral regurgitation assessed by angiography. These patients showed jet areas on Doppler color flow imaging significantly smaller with acute valvular regurgitation (decreased compliant left atrium, extremely increased atrial pressure) as compared with chronic valvular regurgitation (compliant left 


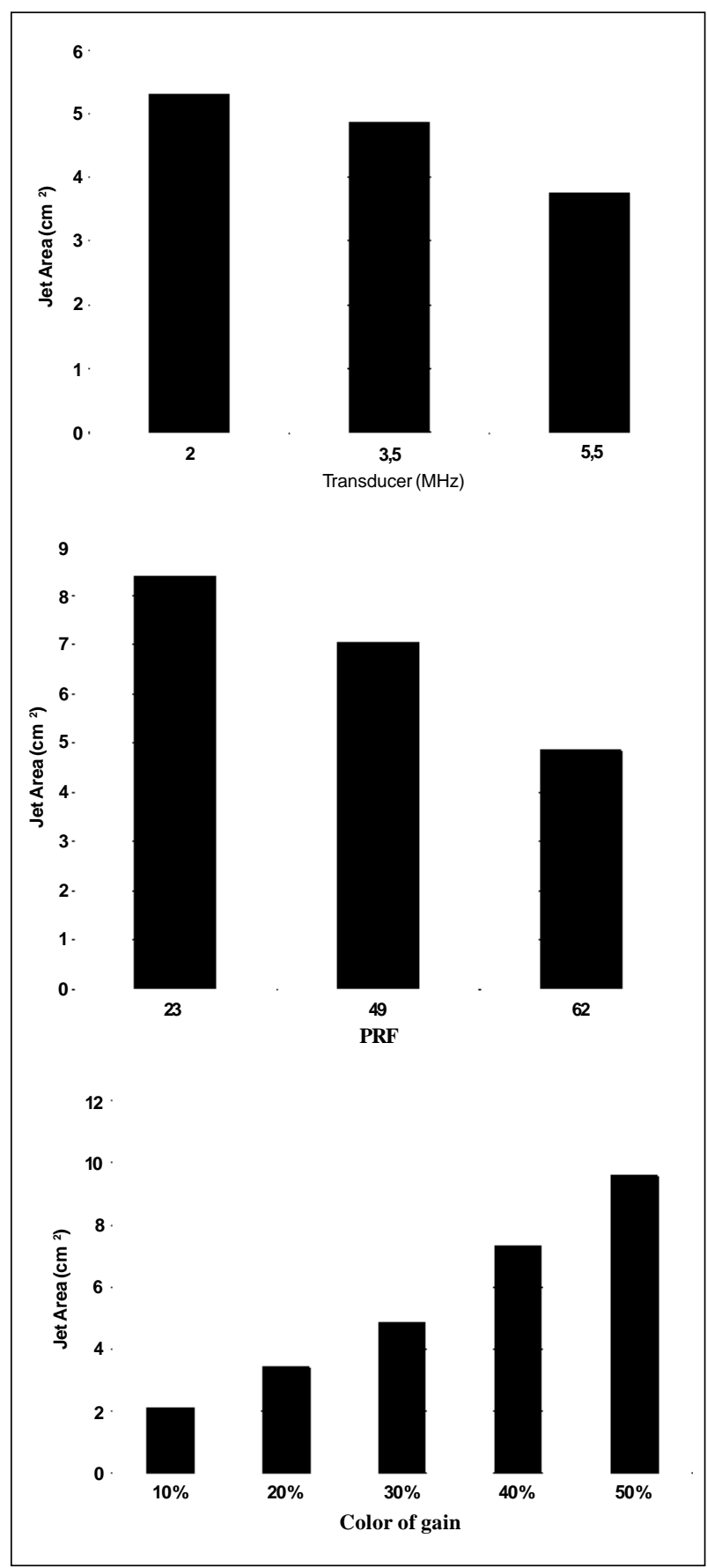

Fig. 1 - Effects of the transducer frequency (higher panel), of the pulse repetition frequency (PRF) (intermediate panel), and of color gain (lower panel) on the regurgitant jet area. Mean values observed in an in vitro experimental model, in which the injecting bomb produced isolated jets through an orifice of known dimensions with injection of a same blood volume at a constant velocity.

atrial cavity, mild to moderate increase in atrial pressure). Yet in the clinical context, variations in regurgitant jet dimensions with the use of maneuvers that increase blood pressure have been reported, with the infusion of angiotensin ${ }^{51}$ or isometric exercise $^{52,53}$. The heart rate has an inverse relation to jet size, and its influence may be minimized by the increase in the frequency of image sampling ${ }^{54}$.

An additional hydrodynamic factor has complicated even more the potential of jet dimension for quantifying the magnitude of valvular regurgitations. Clinical observations have shown that in patients with mitral regurgitation the correlation between the jet area and the regurgitant volume or fraction becomes markedly worse when the jet is eccentric, heading to the atrial wall ${ }^{55}$. When a fluid crosses an orifice with its trajectory directed to the proximity of a wall, incorporation of the liquid located between the jet and the wall occurs due to the increase in the velocity in this area. Therefore, the shear stresses are modified, and great variability in the velocities and reduction in pressure in this area occur, characterizing an effect of the Ventura type, which determines flow deviation in the direction of that wall, and even adhesion of the jet to the wall (Coanda effect) ${ }^{56,57}$. Experimental ${ }^{58}$ and clinical ${ }^{55,59}$ studies have shown that the area of an eccentric jet that adheres to a wall may reach a magnitude equivalent to $40 \%$ of the one observed for a central regurgitant jet corresponding to the same regurgitant volume. Spatial interaction between flows that maintain certain proximity may also have significant clinical implications, especially in patients with aortic regurgitation, in whom the mitral and aortic diastolic flows may have similar directions. In vitro experimental studies ${ }^{60}$ with parallel jets have shown an unequivocal interaction between them, mainly determined by the difference in velocity between the 2 jets.

Regurgitant orifice geometry does not seem to significantly change the spatial distribution of regurgitant jets, because these jets regain their symmetrical shape in a predictable manner in the proximity of the orifice ${ }^{61}$.

If all these difficulties were not enough, conditioned by the multiplicity of the factors that may potentially interfere with the dimensions of these jets, the methodology still has difficulties related to the inter- and intraobserver variability, which in some reports have reached $20 \%{ }^{44,62-64}$. Even a day-to-day variability of $15 \%$ has been reported in a clinical study ${ }^{65}$.

\section{Momentum flux}

The hydrodynamic principle of the conservation of momentum flux has also been employed in an attempt to overcome the difficulties in quantification documented in the analysis of jet dimensions. This variable that expresses the inertia of the jet may be measured by the product of viscosity by the blood flow and velocity or, according to the transformation of the flow into the product of the orifice area by the velocity, this variable may be represented by the product of the orifice area by the square of blood velocity. The momentum will be constant in the entire extension of the central part of the noneccentric regurgitant jet beginning at the orifice. Incorporation of mass (jet entrainment), which occurs during intrusion of the jet into the receiving chamber, determines a reduction in the velocity beginning at the orifice. In experimental conditions, quantification of this variable in 2 different points of the central region of the jet, starting at the orifice, allows determining the dimension of the regurgitant orifice. 
In vitro experimental studies ${ }^{66,67}$ carried out with continuous flow and with pulsed flow confirmed this methodology, showing high correlation levels ( $\mathrm{r}=0.99$ and 0.98 , respectively) with the regurgitant flow. However, at least two important limitations of its application have been identified: the method cannot be applied to eccentric jets and the measurement of velocity in different points of the jet is not easily performed in clinical conditions, especially in jets generated in the left side of the heart, which are associated with very high velocities that cannot be analyzed on pulsed Doppler. The potential of clinical applicability of the method is, therefore, restricted to regurgitations in the right side of the heart, which are associated with conditions of low gradients of pressure and flow. The lack of familiarity with the concept of momentum and, mainly, the technical difficulties pointed out to estimate velocity in different points of the jet has decisively limited the clinical use of this method.

\section{Vena contracta}

A new perspective for quantifying the magnitude of valvular regurgitations has been established with the analysis of the hydrodynamic behavior of a fluid when crossing an orifice: a progressive acceleration of the fluid heading to the orifice has been observed, keeping the laminar pattern and reaching its highest velocity when crossing the orifice. Jet dimension at that moment is smaller than that of the orifice. This region of higher velocity and smaller flow dimension is called vena contracta. Measurement of jet dimension immediately proximal to the regurgitant orifice may represent an estimation of this orifice dimension and, consequently, an additional method for quantifying valvular regurgitation. This possibility was initially confirmed by Switzer et al $^{68}$ in an experimental model of aortic regurgitation, in which the authors observed that the minimum proximal width (i.e., vena contracta) was highly correlated with the regurgitant fraction and the diameter of the orifice. After that, an increasing number of clinical and experimental studies ${ }^{69-75}$ has confirmed the potential of this technique, using either the transesophageal or transthoracic echocardiography in aortic and mitral regurgitation.

In a clinical study with patients in mitral regurgitation, Feshke et al ${ }^{70}$ compared the vena contracta measure with the jet area, using angiography with iodine contrast as a reference. Images with quality good enough to measure vena contracta were obtained in $92 \%$ of cases. In addition, the vena contracta measure had a better correlation with the angiographic classification as compared with jet dimensions, and it showed a smaller overlap between patients in different degrees of regurgitation. In that study, because the sensitivity was $94 \%$ and specificity $91 \%$, the authors reported a good diagnostic value of the vena contracta measure higher than $0.65 \mathrm{~cm}$ for identifying patients with severe mitral regurgitation. Current availability of modern ultrasound equipment capable of generating color flow images with high spatial resolution close to the regurgitant orifice increases the potential of this methodology, which basically depends on a simple linear measure. Even though the influence of various physical, hemodynamic, and technical factors on the dimensions of vena contracta still deserves further investigation, initial data show that the shape of the regurgitant orifice does not significantly influence this technique ${ }^{75}$.

\section{Acceleration of the flow proximal to the orifice}

The physical principle of conservation of mass has served as a base for describing another method of quantification of the regurgitant volume by Recusani et al ${ }^{76}$. When approaching an orifice, any fluid keeps a pattern of laminar flow in the convergence region proximal to the orifice, with velocities that symmetrically increase. Therefore, fluid layers are formed disposed in concentric hemispheres with the same velocity with which they will cross the orifice. Therefore, the flow in each of these hemispheric layers is the same as that crossing the orifice ${ }^{77}$ and can be calculated through the following equation: $\mathrm{Q}=2 \cdot \pi \cdot \mathrm{r}^{2} \cdot \mathrm{V}_{\mathrm{r}}$

Where, Q corresponds to the flow (in cubic centimeters per second), $\mathrm{V}_{\mathrm{r}}$ is the flow velocity (expressed in centimeters per second) from an $r$ distance (in centimeters) from the orifice, corresponding to one of the hemispheric layers of isovelocity and, consequently, to the flow through the orifice.

The Doppler color flow mapping technique has two peculiarities that make it appropriate for the application of this hemodynamic principle in quantifying valvular regurgitations: 1) it allows spatial documentation of flow velocities in the convergence region proximal to the orifice; 2) as it is a technique that abides by the principles of the pulsed Doppler, when a velocity to be recorded surpasses the Nyquist limit, the phenomenon of direction ambiguity (aliasing) occurs. In color flow mapping, this phenomenon is represented by a complete inversion of the color, from blue to red, or vice-versa, depending on the flow direction. This characteristic makes the documentation of a semicircular image in the convergence region proximal to the orifice possible, which occurs exactly when the velocity surpasses that limit and the color change occurs. Thus it is possible to measure the distance between this point of color change and the regurgitant orifice at the same time when the velocity of the aliasing phenomenon is registered by the ultrasound equipment. This method, popularly known as PISA (Proximal Isovelocity Surface Area), also allows estimation of the regurgitant orifice area. Considering that the flow in a given site may be measured by the product between velocity and area, the regurgitant orifice area may be calculated by the division of the flow through the orifice (measured by the PISA method) by the maximum flow velocity through the orifice (obtained by continuous Doppler).

A substantial number of experimental and clinical studies ${ }^{78-80}$ have shown very high levels of correlation with measures of regurgitant flow and fraction and the semiquantitative angiographic grading. These studies have also shown the adequacy of this methodology for calculating flows and orifice dimensions in patients with valvular 
stenoses, congenital heart diseases, and acquired atrial septal defects ${ }^{81-84}$. Instrumental factors, such as gain of the system, wall filters, frequency of image documentation, type of processing of the color flow, as well as shape of the orifice, do not seem to substantially influence flow quantification by this method ${ }^{85}$. This represents a significant advantage in relation to the techniques of assessment that use the measure of jet area. Some problems, however, have also been identified. One of the most difficult aspects of the method is the precise determination of the regurgitant orifice location, which may cause an error in the measurement of the $r$ distance, a variable to the power of $2^{86}$. Another very debated point regards the shape of the proximal flow convergence region, which seems to be better represented by a hemiellipse than by a hemisphere. This straightening of the curves of isovelocity in the proximity of the orifice leads to underestimation of the regurgitant flow calculated with the conventional formula ${ }^{87,88}$. This underestimation, however, seems to be significant only for regurgitant jets generated in low pressure territories, as occurs in valvular regurgitations of the right side of the heart, and, even in this case, it is possible to foretell it and apply a correction factor. Modifications in equipment adjustments ${ }^{89}$ and application of computed algorithms ${ }^{90,91}$ are alternatives proposed for overcoming this limitation without losing the correlation with the actual flow. Use of the one-dimensional color mode has been proposed as a way of increasing precision of the radius measure. However, due to the possibility of displacement of the cursor from the ideal position, this technique has not been extensively applied ${ }^{92}$. Another potential limitation regards variation in the orifice during ventricular systole described by some authors ${ }^{92-94}$ in mitral regurgitation and, particularly, in patients with mitral valvular prolapse. However, the impact of this variation in the orifice dimension does not seem significant in most of the clinical situations ${ }^{95}$. Another problem may arise when the regurgitant orifice is not located in a plane surface but in the extremity of a funneled structure, which impairs the distribution of the concentric layers of isovelocity. Data available ${ }^{96}$, however, show that it is possible to correct this distortion taking into consideration the angle between the central axis of the orifice and the wall of this funneled surface. At any rate, the use of the proximal flow convergence method may have its application limited when the jets have an eccentric origin, creating a zone of distorted acceleration.

Despite these limitations, the proximal flow convergence method has been consolidated as a concrete alternative for quantifying the magnitude of valvular regurgitations in the clinical context. Specifically in mitral regurgita- tion, effective areas of the regurgitant orifice higher than $30 \mathrm{~mm}^{2}$ have been documented with this method in patients with severe lesions. On the other hand, those patients with mild regurgitation have shown areas smaller than $10 \mathrm{~mm}^{291}$.

\section{Transesophageal echocardiography}

The possibility of obtaining Doppler echocardiographic images through transesophageal via has brought up some factors that may have an influence on the quantification of valvular regurgitation. At the same time, in addition to a higher definition, it also provides significant information for elucidating the mechanism causing the regurgitation. An additional advantage of the transesophageal approach relates to the greater easiness in documenting the pulmonary veins, which may also be important in quantifying valvular regurgitations. These qualities are even more evident when the magnitude of valvular regurgitation in patients with valvular prostheses is considered, a situation where the ultrasound window is usually inadequate for obtaining high-definition images ${ }^{97,98}$. Studies carried out in native ${ }^{99}$ and prosthetic ${ }^{100}$ valves comparing different Doppler echocardiographic methods of quantification show that the measure of the jet area, absolute or corrected for the left atrial area, has significant limitations in allowing an adequate separation of the different degrees of severity. These studies also stress the greatest precision of the methods of flow convergence proximal to regurgitant orifices and the measurement of vena contracta. In spite of the clear advantages of the transesophageal method, one cannot forget that the previously cited limitations for each of the quantification techniques, including those related to jet eccentricity, remain valid.

As a whole, these elements show the existence of an extremely varied set of Doppler echocardiographic methods available for quantifying valvular regurgitations. Each of these techniques has its peculiarities and may be influenced by different physical, hemodynamic, and instrumental factors ${ }^{47,101}$. Even though the assessment methods of vena contracta and of flow convergence proximal to regurgitant orifices have some advantages when compared with the others, it seems very clear that separately none of the available techniques meets all the necessary requirements for a precise quantification without significant limitations. Therefore, it is possible to foresee that these methods may be concomitantly employed and, based on a conjunct analysis of information obtained, it will be possible to define a grading score of valvular regurgitation, which will separate more adequately the different severity levels of these lesions. 


\section{References}

1. Carabello BA. Mitral valve disease. In: Yusuf S.(ed.). Evidence Based Cardiology. London: BMJ Books, 1998: 798-810.

2. Rahimtoola SH. Indications for surgery in aortic valve disease. In: Yusuf S. (ed.). Evidence Based Cardiology. London: BMJ Books, 1998: 811-832.

3. Sandler H, Dodge HT, Hay RE, Rackley CE. Quantification of valvular insufficiency in man by angiocardiography. Am Heart J 1963; 65: 501-513.

4. Sellers RD, Levy M.J, Amplatz K, Lillehei CW. Left retrograde cardioangiography in acquired cardiac diseases: technique, indications and interpretations in 700 cases. Am J Cardiol 1964; 14: 437- 451.

5. Nagle RE, WalkerD, Grainger RG. The angiographic assessment of mitral incompetence. Clin Radiol 1968; 19: 154-66.

6. Fifer MA, Grossman W. Measurement of left ventricular volumes, ejection fraction, mass and wall stress. In: Grossman W. (ed.). Cardiac Catheterization and Angiography. Philadelphia: Lea \& Febiger,1986: 282-300.

7. Croft CH, Lipscomb K, Mathis K, et al. Limitations of qualitative angiographic grading in aortic or mitral regurgitation. Am J Cardiol 1984; 53: 1593-8.

8. Pearlman AS, Otto CM. Quantification of valvular regurgitation. Echocardiog 1987; 4: 271-87.

9. Sahn DJ, Maciel BC. Physiological valvular regurgitation.Doppler echocardiography and the potential for iatrogenic heart disease. Circulation 1988; 78: 1075-7.

10. Yoshida K, Yoshikawa J, Shakudo M, et al. Color Doppler evaluation of valvular regurgitation in normal subjects. Circulation 1988; 78: 840-7.

11. Takao S, Miyatake K, Izumi S, et al. Physiological pulmonary regurgitation detected by the Doppler technique and its differential diagnosis. J Am Coll Cardiol 1985; 5: 499

12. Lynch JJ, Sagar KB, Wann LS. Tricuspid regurgitation in normal subjects: prevalence and possible mechanism. J Am Coll Cardiol 1986; 7: 145.

13. Maciel BC, Simpson IA, Valdes-CruzLM, et al. Color flow Doppler mapping studies of "physiologic" pulmonary and tricuspid regurgitation: evidence for true regurgitation as opposed to a valve closing volume. J Am Soc Echocardiogr 1991; 4: 589-97.

14. Smith MD. Evaluation of valvular regurgitation by Doppler echocardiography. Cardiol Clin 1991; 9: 193-228.

15. Akasaka T, Yoshikawa J, Yoshida K, et al. Age related valvular regurgitation: a study by pulsed doppler echo. Circulation 1987; 76: 262-5.

16. Choong CY, Abascal VM, Weyman J, et al. Prevalence of valvular regurgitation by Doppler echocardiography in patients with structurally normal hearts by two-dimensional echocardiography. Am Heart J 1989; 117: 636-42.

17. Klein AL, Burstow DJ, Tajik AJ, et al. Age-related prevalence of valvular regurgitation in normal subjects: a comprehensive color flow examination of 118 volunteers. J Am Soc Echocardiogr 1990; 2: 54-63.

18. Abassi AS, Allen MW, DeCristofaro D, Ungar I. Detection and estimation of the degree of mitral regurgitation by range-gated pulsed Doppler ecocardiography. Circulation 1980;. 61: 143-7.

19. Blumlein S, Bouchard A, Schiller NB, et al. Quantitation of mitral regurgitation by Doppler echocardiography. Circulation 1986; 74: 306-14.

20. Zhang Y, Ihlen H, Myhre E, Levorstad K, Nitter-Hauge S. Measurement of mitral regurgitation by Doppler echocardiography. Br Heart J 1985; 54: 384-91.

21. Ciobanu M, Abbasi AS, Allen M, Hermer A, Spellberg R. Pulsed Doppler echocardiography in the diagnosis and estimation of severity of aortic insufficiency. Am J Cardiol 1982; 49: 339-43.

22. Borras X, Carreras F, Auge JM, Pons-Llado G. Prospective validation of detection and quantitative assessment of chronic aortic regurgitation by a combined echocardiography and Doppler method. J Am Soc Echocardiogr 1988; 1:422-9.

23. Bolger AF, Eigler NL, Maurer G. Quantifying valvular regurgitation: limitations and inherent assumptions of doppler techniques. Circulation 1988; 78: 1316-18.

24. Hatle L, Andersen B. In: Doppler Ultrasound in Cardiology: Physical Principles and Clinical Applications,ed 2. Philadelphia: Lea \& Febiger, 1985.

25. Weyman AE. In: Principles and Practice of Echocardiography ed.2. Philadelphia: Lea \& Febiger, 1994: 432

26. Bolger AF, Eidenvall L, Ask P, Loyd D, Wranne B. Understanding continuoswave Doppler signal intensity as a measure of regurgitant severity. J Am Soc Echocardiogr 1997; 10: 613-22.

27. Castello R, Pearson AC, Lenzen P, Labovitz AJ. Effect of mitral regurgitation on pulmonary venous velocities derived from transesophageal echocardiography color-guided pulsed Doppler imaging. J Am Coll Cardiol 1991; 17: 1499-506.

28. Klein AL, Obarski TP, Stewart WJ, et al Transesophageal Doppler echocardiography of pulmonary venous flow: a new marker of mitral regurgitation severity. J Am Coll Cardiol 1991; 18: 518-26.

29. Seiler C, Aeschbacher BC, Meier B. Quantitation of mitral regurgitation using the systolic/diastolic pulmonary venous flow velocity ratio. J Am Coll Cardiol 1998; 31: 1383-90
30. Labovitz AJ, Ferrara RP, Kern MJ, Bryg RJ, Mrosek DG, Williams GA. Quantitative evaluation of aortic insufficiency by continuous wave Doppler echocardiography. J Am Coll Cardiol 1986; 8:1341-7.

31. Teague SM, Heinsimer JA, Anderson JL, Sublett K, Voyles WF, Thadani U. Quantification of aortic regurgitation utilizing continuous wave Doppler ultrasound. J Am Coll Cardiol 1986; 8: 592-9.

32. Takenaka K, Dabestani A, Gardin JM, etal. A simple Dopplerechocardiographic method for estimating severity of aortic regurgitation. Am J Cardiol 1986; 57: 1340-3.

33. Fisher DC, Sahn DJ, Friedman JM, et al. The mitral valve orifice method for noninvasive two-dimensional echo Doppler determination of cardiac output. Circulation 1983; 67: 872-7.

34. Enriquez-Sarano M., Bailey KR, Seward JB, Tajik AJ, Krohn MJ, Mays JM. Quantitative Doppler assessment of valvular regurgitation. Circulation 1993; 87: 841-8.

35. Omoto R, Yokote Y, Takamoto S, et al. The development of real time two-dimensional Doppler echocardiography and its clinical significance in acquired valvular diseases. With special references to the evaluation of valvular regurgitation. Jpn Heart J 1984; 25: 325-40.

36. Miyatake K, Izumi S, Okamoto M, et al. Semiquantitative grading of severity of mitral regurgitation by real-time two-dimensional Doppler flow imaging technique. J Am Coll Cardiol 1986; 7: 82-8.

37. Mohr-Kahaly S,ErbelR,Zenker G, etal. Semiquantitative grading of mitral regurgitation by color-coded Doppler echocardiography. Int J Cardiol 1989; 23: 223-30.

38. Helmcke F, Nanda NC, Hsiung MC, et al. Color Doppler assessment of mitral regurgitation with orthogonal planes. Circulation 1987; 75: 175-83.

39. Spain MG, Smith MD, Grayburn PA, Harlamert EA, DeMaria AN. Quantitative assessment of mitral regurgitation by Doppler color flow imaging: angiographic and hemodynamic correlations. J Am Coll Cardiol 1989; 13: 585-90.

40. Maciel BC, Moises VA, Shandas R, et al. Effects of pressure and volume of the receiving chamber on the spatial distribution of regurgitant jets as imaged by color doppler flow mapping. An in vitro study. Circulation 1991; 83: 605-13.

41. Galassi AR, Nihoyannopoulos P, Pupita G, Odawara H, Crea F, McKenna WJ. Assessment of color flow imaging in the grading of valvular regurgitation. Eur Heart J 1990; 11: 1101-08.

42. Perry GJ, HelmckeF, Nanda NC, Byard C, Soto B. Evaluation of aortic insufficiency by Doppler color flow mapping. J Am Coll Cardiol 1987; 9: 952-9.

43. Stevenson JG. Two-dimensional color Doppler estimation of the severity of atrioventricular valve regurgitation: important effects of instrument gain setting, pulse repetition frequency, and carrier frequency. J Am Soc Echocardiog 1989;2: 1-10.

44. Hoit BD, Jones M, Eidbo EE, Elias W, Sahn DJ. Sources of variability for Doppler color flow mapping of regurgitant jets in an animal model of mitral regurgitation. J Am Coll Cardiol 1989; 13: 1631-6.

45. Stewart WJ, Cohen GI, Salcedo EE. Doppler color flow image size: dependence on instrument settings. Echocardiography 1991; 8: 319-27.

46. Utsunomiya T, Ogawa T, King W, et al. Pitfalls in the display of color Doppler jet areas: combined variability due to Doppler angle, frame rate, and scanning direction. Echocardiography 1990; 7: 739-45.

47. Sahn DJ. Instrumentation and physical factors related to visualization of stenotic and regurgitant jets by Doppler color flow mapping. J Am Coll Cardiol 1988; 12: $1354-65$.

48. Simpson IA, Valdes-CruzLM, Sahn DJ, Murillo A, Tamura T, Chung KJ. Doppler color flow mapping of simulated in vitro regurgitant jets: evaluation of the effects of orifice size and hemodynamic variables. J Am Coll Cardiol 1989; 13: 1195-207.

49. Losordo DW, Pastore JO, Coletta D, Kenny D, Isner JM. Limitations of color flow Doppler imaging in the quantification of valvular regurgitation: velocity of regurgitant jet, rather than volume, determines size of color Doppler image. Am Heart J 1993; 126: 168-76.

50. Harlamert EA, Smith MD, Spain MG, Gash DL, Berk MR, DeMaria AN. Color Doppler flow imaging underestimates the severity of acute mitral regurgitation. Circulation 1988; 78(suppl 2): 434.

51. BertucciC, Valdes-CruzLM, Recusani F, et al. Doppler study of the effects of afterload on the spatial distribution of mitral regurgitant jets. J Am Coll Cardiol 1987; 9(suppl A): 67A.

52. SaenzCB, Deumite NJ, Roitman DI, Moos S, Nanda NC, Soto B. Limitations of color Doppler in quantitative assessment of mitral regurgitation. Circulation 1985; 72(suppl 3): 99.

53. Spain MG, Smith MD, Kwan OL, DeMaria AN. Effect of isometric exercise on mitral and aortic regurgitation as assessed by color Doppler flow imaging. Am J Cardiol 1990; 65: 78-83.

54. Cape EG, Yoganathan AP, Levine RA. Increased heart rate can cause underestimation of regurgitant jet size by Doppler color flow mapping. J Am Coll Cardiol 1993; 21: 1029-37. 
55. Chen C, Thomas JD, Anconina J, et al. Impact of impinging wall jet on color Doppler quantification of mitral regurgitation. Circulation 1991; 84: 712-20.

56. Cape EG, Yoganathan AP, Weyman AE, Levine RA. Adjacent solid boundaries alter the size of regurgitant jets on Doppler color flow maps. J Am Coll Cardiol 1991; 17: 1094-102.

57. Chao K, Moises VA, Shandas R, Elkadi T, Sahn DJ, Weintraub R. Influence of the Coanda effect on color Doppler jet area and color encoding. In vitro studies using color Doppler flow mapping. Circulation 1992; 85: 333-41.

58. Zhang J, Shiota T, Shandas R, et al. Effects of adjacent surfaces of different shapes on regurgitant jet sizes: an in vitro study using color Doppler imaging and laserilluminated dye visualization. J Am Coll Cardiol 1993; 22: 1522-9.

59. Enriquez-Sarano M, Tajik AJ, Bailey KR, Seward JB. Color flow imaging compared with quantitative Doppler assessment of severity of mitral regurgitation: influence of eccentricity of jet and mechanism of regurgitation. J Am Coll Cardiol 1993; 21: 1211-19.

60. Shandas R, Moises VA, Maciel BC, Sahn DJ. Demonstration of dual parallel jet interaction in an in vitro model of multivalvular disease by use of optical visualization and color Doppler flow mapping. J Am Soc Echocardiogr 1993; 6: 124-33.

61. Thomas JD, O'Shea JP, Rodriguez L, Popovic AD, Svizerro T, Weyman AE. Impact of orifice geometry on the shape of jets: an in vitro Doppler color flow study. J Am Coll Cardiol 1991; 17: 901-08.

62. Castello R, Lenzen P, Aguirre F, Labovitz A. Variability in quatitation of mitral regurgitation by Doppler color flow mapping: comparison of transthoracic and trans esophageal studies. J Am Coll Cardiol, 1992; 20: 433-8.

63. Wong M, Matsumura M, Suzuki K, Omoto R. Technical and biologic sources of variability in the mapping of aortic, mitral and tricuspid color flow jets. Am J Cardiol 1987; 60: 847-51.

64. Willems TP, Steyerberg EW, van Herwerden LA, et al. Reproducibility of color Doppler flow quantification of aortic regurgitation. J Am Soc Echocardiogr 1997; 10: 899-903.

65. Grayburn PA, Pryor SL, Levine BD, Klein M.N, Taylor AL. Day to day variability of Doppler color flow jets in mitral regurgitation. J Am Coll Cardiol 1989; 14: 936-40.

66. Cape EG, Skoufis EG, Weyman AE, Yoganathan AP, Levine RA. A new method for noninvasive quantification of valvular regurgitation based on conservation of momentum. In vitro validation. Circulation 1989; 79: 1343-53.

67. Thomas JD, Liu CM, Flachskampf FA, O'Shea JP, Davidoff R, Weyman AE. Quantification of jet flow by momentum analysis. An in vitro color Doppler flow study. Circulation 1990; 81: 247-59.

68. Switzer DF, Yoganathan AP, Nanda NC, Woo Y-R, Ridgway AJ. Calibration of color Doppler flow mapping during extreme conditions in vitro: a foundation for a reliable quantitative grading system for aortic incompetence. Circulation 1987; 75: 837-46.

69. Tribouilloy C, Shen WF, Quéré JP, et al. Assessment of severity of mitral regurgitation by measuring regurgitant jet width at its origin with transesophageal Doppler color flow imaging. Circulation 1992; 85: 1248-53.

70. Feshke W, Omran H, Manz M, Köhler J, Hagendorff A, Lüderitz B. Color-coded Doppler imaging of the vena contracta as a basis for quantification of pure mitral regurgitation. Am J Cardiol 1994; 73: 268-74

71. Hall AS, Brickner E, Wilett DL, Irani WN, Afridi I, Grayburn PA. Assessment of mitral regurgitation severity by Doppler color flow mapping of the vena contracta. Circulation 1997; 95: 636-42.

72. Zhou X, Jones M, Shiota T, Yamada I, Teien D, Sahn DJ. Vena contracta imaged by Doppler color flow mapping predicts the severity of eccentric mitral regurgitation better than color jet area: a chronic animal study. J Am Coll Cardiol 1997; 30:1393-8.

73. Grayburn PA, Fehske W, Omran H, Brickner ME, Lüderitz B. Multiplane transesophageal echocardiographic assessment of mitral regurgitation by Doppler color flow mapping of the vena contracta. Am J Cardiol 1994; 74: 912-17.

74. Ishii M, Jones M, Shiota T, et al. Quantifying aortic regurgitation by vena contracta imaging: a chronic animal model study. Circulation 1997; 27: 2009-15.

75. Baumgartner H, Schima H, Kuhn P. Value and limitations of proximal jet dimensions for the quantitation of valvular regurgitation: an in vitro study using Doppler flow imaging. J Am Soc Echocardiogr 1991; 4: 57-66.

76. Recusani F, Bargiggia GS, Yoganathan AP, et al. A new method for quantification of regurgitant flow rate using color Doppler flow imaging of the flow convergence region proximal to a discrete orifice. An in vitro study. Circulation 1991; 83: 594-604.

77. Simpson IA, Sahn DJ. Quantification of valvular regurgitation by Doppler echocardiography. Circulation 1991; 84(suppl 1): 188-92..

78. Bargiggia GS, Tronconi L, Sahn DJ, et al. A new method for quantification of mitral regurgitation based on color flow Doppler imaging of flow convergence proximal to regurgitant orifice. Circulation 1991; 84: 1481-9.

79. Giesler M, Grossmann G, Schmidt A, et al. Color Doppler echocardiographic de- termination of mitral regurgitant flow from the proximal velocity profile of the flow convergence region. Am J Cardiol 1993; 71: 217-24.

80. Chen C, Koschyk D, Brockhoff C, et al. Noninvasive estimation of regurgitant flow rate and volume in patients with mitral regurgitation by Doppler color mapping of accelerating flow field. J Am Coll Cardiol 1993; 21: 374-83.

81. Simpson IA, Sahn DJ, Valdes-Cruz LM, et al. Color Doppler flow mapping in patients with coarctation of the aorta:new observations and improved evaluation with color diameter and proximal aceleration as predictors of severity. Circulation 1988; 77: 736-44.

82. Moises VA, Maciel BC, Hornberger LK, et al. A new method for noninvasive estimation of ventricular septal defect shunt flow by Doppler color flow mapping: imaging of laminar flow convergence region on the left septal surface. J Am Coll Cardiol 1991; 18: 824-32.

83. Rittoo D, Sutherland GR, Shaw,TRD. Quantification of left-to-right atrial shunting and defect size after ballon mitral commissurotomy using biplane transesophageal echocardiography, color Doppler mapping, and the principle of proximal flow convergence. Circulation 1993; 87: 1591-603.

84. Rodriguez L, Thomas JD, Monterosso V, et al. Validation of the proximal flow convergence method. Calculation of orifice area in patients with mitral stenosis Circulation 1993; 88: 1157-65.

85. Utsunomiya T, Ogawa T, Doshi R, et al. Doppler color flow “proximal isovelocity surface area" method for estimating volume flow rate: effects of orifice shape and machine factors. J Am Coll Cardiol 1991; 17: 1103-11.

86. Thomas JD, Davidoff R, Cape EG. Fluid dynamics of regurgitant jets and their imaging by color Doppler. In: Weyman AE (ed). Principles and Practice of Echocardiography. Philadelphia: Lea \& Febiger, 1994: 234-55.

87. Barclay AS, Eidenvall L, Karlsson M, et al. The shape of the proximal isovelocity surface area varies with regurgitant orifice size and distance from orifice: computer simulation and model experiments with color M-mode tecnique. J Am Soc Echocardiogr 1993; 6: 433-45.

88. Rodriguez L, Anconina J, Flachskampf FA, Weyman AE, Levine RA. Impact of finite orifice size on proximal flow convergence:implications for Doppler quantification of valvular regurgitation. Circ Res 1992; 88: 1699-708.

89. Utsunomiya T, Doshi R, Patel D, et al. Calculation of volume flow rate by the proximal isovelocity surface area method: simplified approach using color Doppler zero baseline shift. J Am Coll Cardiol 1993; 22: 277-82.

90. Vandervoort PM, Thoreau DH, Rivera JM, Levine RA, Weyman AE, Thomas JD Automated flow rate calculations based on digital analysis of flow convergence proximal to regurgitant orifices. J Am Coll Cardiol 1993; 22: 535-41.

91. Vandervoort PM, Rivera JM, Mele D, et al. Application of color Doppler flow mapping to calculate effective regurgitant orifice area. An in vitro study and initial clinical observations. Circulation 1993; 88: 1150-6.

92. SchwammenthalE, Chen C, Benning F, Block M, Briethardt G, Levine RA. Dynamics of mitral regurgitant flow and orifice area. Physiologic application of the proximal flow convergence method: clinical data and experimental testing. Circulation 1994, 90:307-22.

93. Shiota T, Jones M, Teien DE, et al. Dynamic change in mitral regurgitant orifice area: comparisom of color Doppler echocardiographic and eletromagnetic flowmeterbased methods in a chronic animal model. J Am Coll Cardiol 1995; 26:528-36.

94. Enriquez-Sarano M, Sinak LJ, Tajik J, Bailey KR, Seward JB. Changes in effective regurgitant orifice throughout systole in patients with mitral valve prolapse. A clinical study using the proximal isovelocity surface area method. Circulation 1995; 92: 2951-8.

95. Simpson IA, Shiota T, Gharib M, Sahn DJ. Current status of flow convergence for clinical applications: is it a leaning tower of "PISA"? J Am Coll Cardiol 1996; 27:504-09.

96. Rodriguez L, Thomas JD, Monterosso V, et al. Validadtion of the proximal flow convergence method. Calculation of orifice area in patients with mitral stenosis Circulation 1991; 88: 1157-65.

97. Yoganathan AP, Cape EG, Sung HW, Williams FP, Jimoh AV. Review of hydrodynamic principles for the cardiologist: applications to the study of blood flow and jets by imaging techniques. J Am Coll Cardiol 1988; 12: 1344-53.

98. Olmos L, Salazar G, Barbetseas J, Quinones MA, Zoghbi WA. Usefulness of transthoracic echocardiography in tetecting dignificant prosthetic mitral valve regurgitation. Am J Cardiol 1999; 83: 199-205.

99. Flachskampf FA, Frieske R, Engelhard B, et al. Comparison of transesophageal Doppler methods with angiography for evaluation of thr severity of mitral regurgitation. J Am Soc Echocardiogr 1998; 11: 882-92.

100. Fischer $\mathrm{CH}, \mathrm{Campos} \mathrm{F}^{\circ} \mathrm{O}$, Moises VA, et al.Quantificação de insuficiência protética mitral por diferentes métodos de avaliação pela ecocardiografia transesofágica. Arq Bras Cardiol 1998; 71: 741-5.

101. Yoganathan AP, Cape EG, Sung HW, Williams FP, Jimoh AV. Review of hydrodynamic principles for the cardiologist: Applications to the study of blood flow and jets by imaging techniques. J Am Coll Cardiol 1988; 12: 1344: 53. 